Pacific Journal of Mathematics

CLASSIFICATION OF ESSENTIAL COMMUTANTS OF 


\title{
CLASSIFICATION OF ESSENTIAL COMMUTANTS OF ABELIAN VON NEUMANN ALGEBRAS
}

\author{
BRUCE H. WAGNER
}

\begin{abstract}
The main purpose of this paper is to classify the $C^{*}$-algebras of the form $\mathfrak{A}^{\prime}+\mathscr{K}$, where $\mathfrak{A}^{\prime}$ denotes the commutant of an abelian von Neumann algebra $\mathfrak{A}$, and $\mathscr{K}$ is the set of compact operators. By the famous result of Johnson and Parrott, $\mathfrak{A}^{\prime}+\mathscr{K}$ is the same as the essential commutant of $\mathfrak{A}$. These algebras were studied by Plastiras in the special case in which $\mathfrak{A}$ is generated by its minimal projections and in addition all of these projections are finite dimensional. Using a theorem of Andersen, we are able to generalize Plastiras' main results to general abelian von Neumann algebras. We also study the automorphism groups and derivations of these algebras.
\end{abstract}

If $\mathfrak{A}$ is an abelian von Neumann algebra, then its projection lattice $\mathscr{L}$ is a complemented commutative subspace lattice, and of course $\mathfrak{A}^{\prime}=\mathscr{L}^{\prime}$. Since our results are given in terms of the lattice, we simply start with such a lattice $\mathscr{L}$ and consider the algebra $\mathscr{L}^{\prime}+\mathscr{K}$. In Corollaries 6 and 9, we give necessary and sufficient conditions for two such algebras $\mathscr{L}^{\prime}+\mathscr{H}$ and $\mathscr{M}^{\prime}+\mathscr{K}$ to be equal or isomorphic. We then turn to automorphisms, and first catagorize those algebras for which every unitary operator implementing an automorphism splits (Theorem 11), and then determine those algebras for which every automorphism is inner (Theorem 12). These four results generalize the most important results in [P]. We next calculate the outer automorphism group (Corollary 20), and finally show that every derivation of such an algebra is inner (Theorem 22). This latter portion of the paper was motivated primarily by similar studies with nests, namely [W1], [W2], and [DW].

All Hilbert spaces in this paper will be separable and infinite dimensional, and will usually be denoted by $\mathscr{H} \cdot \mathscr{B}(\mathscr{H})$ will be used to denote the set of bounded operators on $\mathscr{H}$, and the set of compact operators will be given by $\mathscr{K}(\mathscr{H})$, or just $\mathscr{K}$ if the Hilbert space is clear from the context. If $\mathscr{S} \subseteq \mathscr{B}(\mathscr{H})$, then $\mathscr{S}^{\prime}=\{T \in \mathscr{B}(\mathscr{H}): T S=$ $S T$ for all $S \in \mathscr{S}\}$ is the commutant of $\mathscr{S}$, and $\mathscr{S}^{\prime \prime}$ denotes the double commutant $\left(\mathscr{S}^{\prime}\right)^{\prime}$ of $\mathscr{S}$. The essential commutant of $\mathscr{S}$ is $\{T \in \mathscr{B}(\mathscr{H}): T S-S T \in \mathscr{K}$ for all $S \in \mathscr{S}\}$. All projections on Hilbert space will be self-adjoint. 
A commutative subspace lattice (CSL) is a commuting set of projections on $\mathscr{H}$ which is closed under the lattice operations $P \vee Q=$ $P+Q-P Q$ and $P \wedge Q=P Q$, is closed in the strong operator topology, and contains 0 and $I$. A projection $E \in \mathscr{L}$ is an atom if $E P=0$ or $E$ for all $P \in \mathscr{L}$. Note that all atoms are mutually orthogonal. $\mathscr{L}$ is nonatomic, or continuous, if it has no atoms, and purely atomic if $\sum E_{i}=I$, where the sum is taken over all atoms $E_{i}$ of $\mathscr{L}$ and convergence is in the strong operator topology. As is often done in the study of commutative subspace lattices, we will sometimes blur the distinction between a projection $P$ and its range $P \mathscr{H}$. In this spirit, we will use $\operatorname{dim} P$ to denote the dimension of $P \mathscr{H}$. Also, a projection is infinite or finite if its range is infinite or finite dimensional, respectively. Define $\mathscr{A}(\mathscr{L})=\{$ atoms of $\mathscr{L}\}, \mathscr{I}(\mathscr{L})=\{$ infinite atoms of $\mathscr{L}\}$, and $\mathscr{F}(\mathscr{L})=\{$ finite atoms of $\mathscr{L}\}$, and let $i(\mathscr{L})$ denote the cardinality of $\mathscr{I}(\mathscr{L})$. Let $P_{a}=\sum_{A \in \mathscr{A}(\mathscr{L})} A$ and define $P_{c}=I-P_{a}$. Then $\mathscr{L}_{a} \subseteq \mathscr{B}\left(P_{a} \mathscr{H}\right)$ is defined to be the purely atomic CSL $\left.\mathscr{L}\right|_{P_{a} \mathscr{L}}$ and likewise $\mathscr{L}_{c} \subseteq \mathscr{B}\left(P_{c} \mathscr{H}\right)$ is the nonatomic CSL $\left.\mathscr{L}\right|_{P_{c} \mathscr{H}}$. Finally, we define the operator $\delta_{\mathscr{L}}$ by $\delta_{\mathscr{L}}(T)=\sum E_{i} T E_{i}$, where again the sum is taken over all atoms in $\mathscr{L}$ and convergence is in the strong operator topology. If $\mathscr{L}$ is purely atomic, then $T \in \mathscr{L}^{\prime}$ if and only if $T=\delta_{\mathscr{L}}(T)$, and $T \in \mathscr{L}^{\prime}+\mathscr{K}$ if and only if $T-\delta_{\mathscr{L}}(T) \in \mathscr{K}$ by [W3, Lemma 5] (the proof given in [W3] is for a special case, but it works for the general case as well).

If $V$ is a unitary operator, then $\operatorname{Ad} V$ is the operator defined by $(\operatorname{Ad} V)(A)=V A V^{*}$. An isomorphism $\alpha$ of two $C^{*}$-algebras $\mathfrak{A}$ and $\mathfrak{B}$ is spatial if $\alpha=\operatorname{Ad} V$ for some $V$. Let $\operatorname{Aut}(\mathfrak{A})=\{$ automorphisms of $\mathfrak{A}\}$ and $\operatorname{Inn}(\mathfrak{A})=\{$ inner automorphisms of $\mathfrak{A}\}=\{\operatorname{Ad} V: V \in \mathfrak{A}\}$, and let the outer automorphism group $\operatorname{Aut}(\mathfrak{A}) / \operatorname{Inn}(\mathfrak{A})$ be denoted by $\operatorname{Out}(\mathfrak{A})$. In addition, we will use $s-\operatorname{Aut}(\mathfrak{A})$ to represent the group of spatial automorphisms of $\mathfrak{A}$. The notation $\cong$ will be used for both unitary equivalence and group isomorphism as appropriate.

A CSL $\mathscr{L}$ is a nest if it is linearly ordered by the usual ordering of range inclusion, and it is complemented if $P^{\perp} \in \mathscr{L}$ for all $P \in \mathscr{L}$. A complemented CSL is the same as a complete Boolean algebra of projections, and the projection lattice of every abelian von Neumann algebra is a complemented CSL. On the other hand, if $\mathscr{L}$ is a CSL, then $\mathscr{L}^{\prime \prime}$ is an abelian von Neumann algebra whose projection lattice is the complemented subspace lattice $\mathscr{C}(\mathscr{L})$ generated by $\mathscr{L}$. Our focus in this paper is the $C^{*}$-algebra $\left(\mathscr{L}^{\prime \prime}\right)^{\prime}+\mathscr{K}=(\mathscr{C}(\mathscr{L}))^{\prime}+\mathscr{K}=$ $\mathscr{L}^{\prime}+\mathscr{K}$, and although this work was motivated by the study of nests, our results will be stated in terms of the projection lattice $\mathscr{C}(\mathscr{L})$ of 
$\mathscr{L}^{\prime \prime}$. Thus, one can start with a nest $\mathscr{L}$ or any other type of CSL, and simply replace $\mathscr{L}$ by $\mathscr{C}(\mathscr{L})$. This will be a common practice in the sequel. $\mathscr{L}^{\prime}+\mathscr{K}$ is the essential commutant of $\mathscr{L}^{\prime \prime}$ by [JP, Theorem 2.1 , so one can also view this as the study of essential commutants of abelian von Neumann algebras.

We first mention two well-known results which we will use later.

LeMMA 1. If $\mathscr{L}$ is nonatomic CSL, then $\mathscr{L}^{\prime} \cap \mathscr{K}=\{0\}$.

Proof. By [Ar, p. 482], there is a continuous nest $\mathscr{M} \subseteq \mathscr{L}$ such that $\mathscr{M}^{\prime}=\mathscr{L}^{\prime}$. The proof can then be completed by applying the argument given in [W3, Theorem 14] to $\mathscr{M}$.

LEMMA 2. If $\mathscr{L}$ and $\mathscr{M}$ are CSL's and $\alpha$ is a $C^{*}$-isomorphism of $\mathscr{L}^{\prime}+\mathscr{K}$ onto $\mathscr{M}^{\prime}+\mathscr{K}$, then $\alpha$ is spatial.

Proof. The result follows by a slight modification of the argument given in [JP, Lemma 4.5].

Definition 3. Suppose $\mathscr{L}$ is a complemented CSL and $P$ is a finite projection in $\mathscr{L}^{\prime}$. We define $\mathscr{L}^{P}$ to be the complemented CSL generated by $P$ and $\left\{P^{\perp} L: L \in \mathscr{L}\right\}$, and we say that $\mathscr{L}^{P}$ is a finite perturbation of $\mathscr{L}$ (this usage differs slightly from that in [D2] and [DW], but the idea is the same). Note that $\left(\mathscr{L}^{P}\right)^{\prime}+\mathscr{K}=\mathscr{L}^{\prime}+\mathscr{K}$. Also, $P \leq P_{a}$ by Lemma 1 , and therefore $\mathscr{L}_{c}^{P}=\mathscr{L}_{c}$.

Our main tool for analyzing isomorphisms and automorphisms of $\mathscr{L}^{\prime}+\mathscr{K}$ is the following theorem of Andersen.

Theorem 4 [An, Proposition 2.3.3]. If $\mathscr{L}$ and $\mathscr{M}$ are complemented CSL's and $\mathscr{L}^{\prime}+\mathscr{K}=\mathscr{M}^{\prime}+\mathscr{K}$, then there are finite projections $P \in \mathscr{L}^{\prime}, Q \in \mathscr{M}^{\prime}$, and a unitary $U$ with $U-I \in \mathscr{K}$ such that $U \mathscr{L}^{P} U^{*}=\mathscr{M}^{Q}$ and $U P U^{*}=Q$.

It follows that $U\left(\mathscr{L}^{P}\right)^{\prime \prime} U^{*}=\left(\mathscr{M}^{Q}\right)^{\prime \prime}$ and $U\left(\mathscr{L}^{P}\right)^{\prime} U^{*}=\left(\mathscr{M}^{Q}\right)^{\prime}$.

Lemma 5. The projections $P$ and $Q$ in Theorem 4 can be chosen to be sums of finite atoms and subprojections of infinite atoms.

Proof. This lemma is very similar to [DW, Lemma 3.3]. Let $P_{0} \in$ $\mathscr{L}^{\prime}, Q_{0} \in \mathscr{M}^{\prime}$, and $U=I+$ compact be given by Theorem 4 so 
that $U\left(\mathscr{L}^{P_{0}}\right) U^{*}=\mathscr{M}^{Q_{0}}$ and $U P_{0} U^{*}=Q_{0}$. We may write $P_{0}=$ $\sum_{i=1}^{m} A_{i}+A$ and $Q_{0}=\sum_{j=1}^{n} B_{j}+B$, where each $A_{i}\left(B_{j}\right)$ is dominated by a finite atom $E_{i}\left(F_{j}\right)$ and $A$ and $B$ are each dominated by a sum of infinite atoms. Then $E_{i} A_{i}^{\perp}$ and $F_{j} B_{j}^{\perp}$ are finite atoms of $\mathscr{L}^{P_{0}}$ and $\mathscr{M}^{Q_{0}}$, respectively. Let $C_{i}=E_{i} A_{i}^{\perp}, i=1, \ldots, m$, and $C_{j+m}=$ $U^{*}\left(F_{j} B_{j}^{\perp}\right) U, j=1, \ldots, n$. Setting $P=P_{0}+\sum_{i=1}^{m+n} C_{i}$ and $Q=$ $Q_{0}+\sum_{i=1}^{m+n} U C_{i} U^{*}$, it follows easily that $P$ and $Q$ have the desired form, $U\left(\mathscr{L}^{P}\right) U^{*}=\mathscr{M}^{Q}$, and $U P U^{*}=Q$.

For convenience, we will say that a finite projection which is a sum of finite atoms and subprojections of infinite atoms is $\sigma$-finite. We can now characterize when two such algebras $\mathscr{L}^{\prime}+\mathscr{K}$ and $\mathscr{M}^{\prime}+\mathscr{K}$ are equal or isomorphic.

CoRollary 6. Suppose $\mathscr{L}$ and $\mathscr{M}$ are complemented CSL's. Then $\mathscr{L}^{\prime}+\mathscr{K}=\mathscr{M}^{\prime}+\mathscr{K}$ iff there is a unitary $U$ with $U-I \in \mathscr{K}$ and $\sigma$-finite projections $P \in \mathscr{L}^{\prime}$ and $Q \in \mathscr{M}^{\prime}$ such that

(i) $U \mathscr{L}_{c} U^{*}=\mathscr{M}_{c}$, and

(ii) $U \mathscr{L}_{a}^{P} U^{*}=\mathscr{M}_{a}^{Q}$ with $U P U^{*}=Q$.

Proof. Necessity follows immediately from Theorem 4 and Lemma 5. On the other hand, given $U, P$, and $Q$ satisfying (i) and (ii), we have $U\left(\mathscr{L}^{P}\right) U^{*}=\mathscr{M}^{Q}$ since $\mathscr{L}_{c}^{P}=\mathscr{L}_{c}$ and $\mathscr{M}_{c}^{Q}=\mathscr{M}_{c}$. Then $\left(\mathscr{M}^{Q}\right)^{\prime}=U\left(\mathscr{L}^{P}\right)^{\prime} U^{*} \subseteq\left(\mathscr{L}^{P}\right)^{\prime}+\mathscr{K}$ and $\left(\mathscr{L}^{P}\right)^{\prime}=U^{*}\left(\mathscr{M}^{Q}\right)^{\prime} U \subseteq\left(\mathscr{M}^{Q}\right)^{\prime}+$ $\mathscr{K}$ since $U-I \in \mathscr{K}$. The result now follows because $\left(\mathscr{L}^{P}\right)^{\prime}+\mathscr{K}=$ $\mathscr{L}^{\prime}+\mathscr{K}$ and $\left(\mathscr{M}^{Q}\right)^{\prime}+\mathscr{K}=\mathscr{M}^{\prime}+\mathscr{K}$.

If $\mathscr{L}$ and $\mathscr{M}$ are purely atomic with no infinite atoms, then [P, Corollary 14(i)(ii)] follows immediately.

Corollary 7 [P, Corollary 14]. Suppose $\mathscr{L}$ and $\mathscr{M}$ are purely atomic CSL's with atoms $\left\{E_{i}: 1 \leq i<\infty\right\}$ and $\left\{F_{i}: 1 \leq i<\infty\right\}$, respectively, such that $\operatorname{dim} E_{i}, \operatorname{dim} F_{i}<\infty$ for all $i$. Then $\mathscr{L}^{\prime}+\mathscr{H}=$ $\mathscr{M}^{\prime}+\mathscr{K}$ iff there is a unitary $U$ with $U-I \in \mathscr{K}$, finite subsets $N_{0}, N_{1} \subseteq \mathbb{N}$, and a bijection $\pi: \mathbb{N} \backslash N_{0} \rightarrow \mathbb{N} \backslash N_{1}$ such that $U E_{i} U^{*}=$ $E_{\pi(i)}$ for all $i \in \mathbb{N} \backslash N_{0}$ and $U\left(\sum_{i \in N_{0}} E_{i}\right) U^{*}=\sum_{j \in N_{1}} F_{j}$.

Proof. Simply apply Corollary 6 to the complemented lattices generated by $\mathscr{L}$ and $\mathscr{M}$, letting $P=\sum_{i \in N_{0}} E_{i}$ and $Q=\sum_{j \in N_{1}} F_{j}$. 
LemMA 8. Suppose $\mathscr{L}$ and $\mathscr{M}$ are complemented CSL's and $V$ is a unitary operator such that $V\left(\mathscr{L}^{\prime}+\mathscr{K}\right) V^{*}=\mathscr{M}^{\prime}+\mathscr{K}$. Then there is a unitary $U$ with $U-I \in \mathscr{K}$ and $\sigma$-finite projections $P \in \mathscr{L}^{\prime}, Q \in \mathscr{M}^{\prime}$ such that

(i) $(\operatorname{Ad} U V)\left(\mathscr{L}_{c}\right)=\mathscr{M}_{c}$, and

(ii) $(\operatorname{Ad} U V)\left(\mathscr{L}_{a}^{P}\right)=\mathscr{M}_{a}^{Q}$ with $(\operatorname{Ad} U V)(P)=Q$.

Proof. $\mathscr{M}^{\prime}+\mathscr{K}=V\left(\mathscr{L}^{\prime}+\mathscr{K}\right) V^{*}=V \mathscr{L}^{\prime} V^{*}+\mathscr{K}=\left(V \mathscr{L} V^{*}\right)^{\prime}+\mathscr{K}$, so Corollary 6 can be applied to $\mathscr{M}$ and $V \mathscr{L} V^{*}$. Thus, there is a unitary $U$ with $U-I \in \mathscr{H}$ and $\sigma$-finite projections $R \in\left(V \mathscr{L} V^{*}\right)^{\prime}$ and $Q \in \mathscr{M}^{\prime}$ such that $U\left(V \mathscr{L} V^{*}\right)_{c} U^{*}=\mathscr{M}_{c}$ and $U\left(\left(V \mathscr{L} V^{*}\right)_{a}^{R}\right) U^{*}=\mathscr{M}_{a}^{Q}$ with $U R U^{*}=Q$. Now $\left(V \mathscr{L} V^{*}\right)_{c}=V \mathscr{L}_{c} V^{*}$, so $(\operatorname{Ad} U V)\left(\mathscr{L}_{c}\right)=\mathscr{M}_{c}$. Also, $\left(V \mathscr{L} V^{*}\right)_{a}^{R}=V \mathscr{L}_{a}^{V^{*} R V} V^{*}$, so $($ Ad $U V)\left(\mathscr{L}_{a}^{V^{*} R V}\right)=\mathscr{M}_{a}^{Q}$ with $(U V) V^{*} R V(U V)^{*}=Q$. Since $V^{*} R V$ is a $\sigma$-finite projection in $\mathscr{L}^{\prime}$, the result follows by letting $P=V R V^{*}$.

By Lemma 2, $C^{*}$-isomorphism is the same as unitary equivalence for essential commutants of abelian von Neumann algebras, so the next corollary specifies when two such algebras are isomorphic.

CoRollary 9. Suppose $\mathscr{L}$ and $\mathscr{M}$ are complemented CSL's. Then $\mathscr{L}^{\prime}+\mathscr{K} \cong \mathscr{M}^{\prime}+\mathscr{K}$ iff

(i) $\mathscr{L}_{c} \cong \mathscr{M}_{c}$,

(ii) $i(\mathscr{L})=i(\mathscr{M})$, and

(iii) there are finite subsets $\mathscr{P} \subseteq \mathscr{F}(\mathscr{L})$ and $\mathscr{Q} \subseteq \mathscr{F}(\mathscr{M})$ and a bijection $\tau: \mathscr{F}(\mathscr{L}) \backslash \mathscr{P} \rightarrow \mathscr{F}(\mathscr{M}) \backslash \mathscr{Q}$ such that $\operatorname{dim} A=\operatorname{dim} \tau(A)$ for all $A \in \mathscr{F}(\mathscr{L}) \backslash \mathscr{P}$. In addition, if $i(\mathscr{L})=i(\mathscr{M})=0$, then $\operatorname{dim}\left(\sum_{A \in \mathscr{P}} A\right)=\operatorname{dim}\left(\sum_{B \in \mathscr{Q}} B\right)$.

Proof. Suppose $V$ is a unitary operator such that $V\left(\mathscr{L}^{\prime}+\mathscr{K}\right) V^{*}=$ $\mathscr{M}^{\prime}+\mathscr{K}$. By Lemma 8 , there is a unitary $U$ with $U-I \in \mathscr{K}$ and $\sigma$-finite projections $P \in \mathscr{L}^{\prime}, Q \in \mathscr{M}^{\prime}$ such that $(\operatorname{Ad} U V)\left(\mathscr{L}_{c}\right)=\mathscr{M}_{c}$, $(\operatorname{Ad} U V)\left(\mathscr{L}_{a}^{P}\right)=\mathscr{M}_{a}^{Q}$, and $(\operatorname{Ad} U V)(P)=Q$. Then $\mathscr{L}_{c} \cong \mathscr{M}_{c}$ via

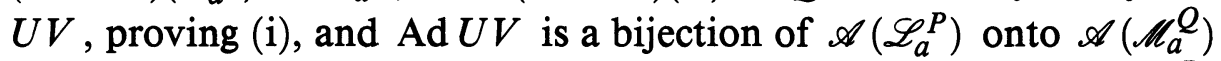
such that $(\operatorname{Ad} U V)(P)=Q$. (ii) now follows since $i(\mathscr{L})=i\left(\mathscr{L}_{a}^{P}\right)$ and $i(\mathscr{M})=i\left(\mathscr{M}_{a}^{Q}\right)$. Finally, let $\mathscr{P}=\{A \in \mathscr{A}(\mathscr{L}): A \leq P\}$ and $\mathscr{Q}=\{A \in \mathscr{A}(\mathscr{M}): A \leq Q\}$. Then $\mathscr{F}\left(\mathscr{L}^{P}\right)=\{P\} \cup(\mathscr{F}(\mathscr{L}) \backslash \mathscr{P})$ and $\mathscr{F}\left(\mathscr{M}^{Q}\right)=\{Q\} \cup(\mathscr{F}(\mathscr{M}) \backslash \mathscr{Q})$. Now just let $\tau=(\operatorname{Ad} U V) \mid \mathscr{F}(\mathscr{L}) \backslash \mathscr{P}$, and (iii) follows (note that if $i(\mathscr{L})=0=i(\mathscr{M})$, then $P=\sum_{A \in \mathscr{P}} A$ and $Q=\sum_{B \in \mathscr{Q}} B$ ). 
Conversely, suppose (i)-(iii) hold. If $\mathscr{L} \subseteq \mathscr{B}\left(\mathscr{H}_{1}\right)$ and $\mathscr{M} \subseteq$ $\mathscr{B}\left(\mathscr{H}_{2}\right)$, let $W: \mathscr{H}_{1} \rightarrow \mathscr{H}_{2}$ be a partial isometry such that $W \mathscr{L}_{c} W^{*}=$ $\mathscr{M}_{c}$. Let $\left\{I_{i}: 1 \leq i \leq i(\mathscr{L})\right\}$ and $\left\{J_{i}: 1 \leq i \leq i(\mathscr{M})=i(\mathscr{L})\right\}$ be enumerations of $\mathscr{I}(\mathscr{L})$ and $\mathscr{I}(\mathscr{M})$, respectively. Let $X: \mathscr{H}_{1} \rightarrow \mathscr{H}_{2}$ be a partial isometry such that $X A X^{*}=\tau(A)$ for all $A \in \mathscr{F}(\mathscr{L}) \backslash \mathscr{P}$. Now if $i(\mathscr{L})=i(\mathscr{M})=0$, let $Y: \mathscr{H}_{1} \rightarrow \mathscr{H}_{2}$ be a partial isometry such that $Y\left(\sum_{A \in \mathscr{P}} A\right) Y^{*}=\sum_{B \in \mathscr{Q}} B$, and define $V=W+X+Y$. Otherwise, let $m=\operatorname{dim}\left(\sum_{A \in \mathscr{P}} A\right)-\operatorname{dim}\left(\sum_{B \in \mathscr{Q}} B\right)$. If $m<0$, let $C \leq I_{1}$ be a projection of dimension $|m|$, and let $D=0$. If $m>0$, let $D \leq J_{1}$ be a projection of dimension $m$, and let $C=0$. If $m=0$, let $C=D=0$. Define $P=C+\sum_{A \in \mathscr{P}} A$ and $Q=D+\sum_{B \in \mathscr{Q}} B$. Now define $Y: \mathscr{H}_{1} \rightarrow \mathscr{H}_{2}$ to be a partial isometry such that $Y P Y^{*}=Q$, $Y I_{i} Y^{*}=J_{i}$ for all $i \geq 2$, and $Y\left(I_{1}-C\right) Y^{*}=J_{1}-D$, and define $V=W+X+Y$. Then in either case $V \mathscr{L}^{P} V^{*}=\mathscr{M}^{Q}$, and it follows that $V\left(\mathscr{L}^{P}\right)^{\prime} V^{*}=\left(\mathscr{M}^{Q}\right)^{\prime}$ and therefore $V\left(\mathscr{L}^{\prime}+\mathscr{K}\right) V^{*}=\mathscr{M}^{\prime}+\mathscr{K}$.

If $\mathscr{L}$ or $\mathscr{M}$ is not complemented, then the same result holds with item (i) changed to $\mathscr{C}\left(\mathscr{L}_{c}\right) \cong \mathscr{C}\left(\mathscr{M}_{c}\right)$, simply by applying the corollary to $\mathscr{C}(\mathscr{L})$ and $\mathscr{C}(\mathscr{M})$. Also, the result for the special case considered in [P] again follows immediately.

Corollary 10 [P, Theorem 1]. Suppose $\mathscr{L}$ and $\mathscr{M}$ are purely atomic CSL's with atoms $\left\{E_{i}: 1 \leq i<\infty\right\}$ and $\left\{F_{i}: 1 \leq i<\infty\right\}$, respectively, such that $\operatorname{dim} E_{i}, \operatorname{dim} F_{i}<\infty$ for all $i$. Then $\mathscr{L}^{\prime}+\mathscr{K} \cong \mathscr{M}^{\prime}+\mathscr{K}$ iff there are finite subsets $N_{0}, N_{1} \subseteq \mathbb{N}$ and a bijection $\pi: \mathbb{N} \backslash N_{0} \rightarrow \mathbb{N} \backslash N_{1}$ such that $\operatorname{dim} E_{i}=\operatorname{dim} E_{\pi(i)}$ for all $i \in \mathbb{N} \backslash N_{0}$ and $\operatorname{dim}\left(\sum_{i \in N_{0}} E_{i}\right)=\operatorname{dim}\left(\sum_{j \in N_{1}} F_{j}\right)$.

Corollary 9 shows that isomorphism is determined by simple conditions on the atoms and unitary equivalence of the nonatomic parts of $\mathscr{L}$ and $\mathscr{M}$. But the latter is equivalent to unitary equivalence of the nonatomic von Neumann algebras $\mathscr{L}_{c}^{\prime \prime}$ and $\mathscr{M}_{c}^{\prime \prime}$. Let $\mathscr{H}_{c}=$ $L^{2}([0,1], \mathscr{B}, \mu)$, where $\mathscr{B}$ is the $\sigma$-algebra of Borel sets and $\mu$ is Lebesgue measure. If $f \in L^{\infty}([0,1], \mathscr{B}, \mu)$, let $M_{f}$ be the multiplication operator acting on $\mathscr{H}_{c}$. For $1 \leq m \leq \infty$, define $\mathscr{Z}^{(m)}=$ $\left\{M_{f} \oplus \cdots \oplus M_{f}: f \in L^{\infty}([0,1], \mathscr{B}, \mu)\right\}$ acting on $\mathscr{H}_{c}^{(m)}$, the direct sum of $m$ copies of $\mathscr{H}_{c}$. Then each nonatomic abelian von Neumann algebra $\mathfrak{A}$ is unitarily equivalent to $\sum_{m \in \mathscr{J}}^{\oplus} \mathscr{Z}^{(m)}$, where $\mathscr{J}$ is a uniquely determined subset of $\{1,2, \ldots, \infty\}$ [KR, §9.4]. We will call $\mathscr{J}$ the multiplicity sequence of $\mathfrak{A}$. Thus, $\mathscr{L}_{c} \cong \mathscr{M}_{c}$ if and only if $\mathscr{L}_{c}^{\prime \prime}$ and $\mathscr{M}_{c}^{\prime \prime}$ have the same multiplicity sequences. 
Another result in $[\mathrm{P}]$ characterizes certain lattices $\mathscr{L}$ for which every unitary $V$ implementing an automorphism of $\mathscr{L}^{\prime}+\mathscr{K}$ splits. This means that $V=W+K$ where $W$ is a unitary satisfying $W \mathscr{L}^{\prime} W^{*}=$ $\mathscr{L}^{\prime}$ and $K \in \mathscr{K}$. Equivalently, $V=W_{1} W_{2}$ for some unitaries $W_{i}$ satisfying $W_{1} \mathscr{L}^{\prime} W_{1}^{*}=\mathscr{L}^{\prime}$ and $W_{2}-I \in \mathscr{K}$ (just let $W_{1}=W$ and $\left.W_{2}=W^{*} V\right)$. If $\mathscr{L}$ is a multiplicity-free CSL $\left(\mathscr{L}^{\prime}=\mathscr{L}^{\prime \prime}\right.$ is maximal abelian), then every such unitary splits [JP, Theorem 4.10]. However, as shown below, there are also other cases for which this is true. In the following proof, ind $(T)$ denotes the Fredholm index of an operator $T$.

Theorem 11. Let $\mathscr{L}$ be a CSL. Define

$$
c(k)=\operatorname{card}\{A \in \mathscr{A}(\mathscr{L}): \operatorname{dim} A=k\} .
$$

Then every unitary which implements an automorphism of $\mathscr{L}^{\prime}+\mathscr{K}$ splits if and only if either

(i) $c(\infty)=0$ and $c(k)=\infty$ for at most one $k$, or

(ii) $c(\infty)=1$ and $c(k)<\infty$ for all $k$.

Proof. By replacing $\mathscr{L}$ with the complemented lattice generated by $\mathscr{L}$, we can assume without loss of generality that $\mathscr{L}$ is complemented. Suppose (i) holds, and suppose that $V$ is a unitary satisfying $V\left(\mathscr{L}^{\prime}+\mathscr{K}\right) V^{*}=\mathscr{L}^{\prime}+\mathscr{K}$. Then by Lemma 8 , there is a unitary $U$ with $U-I \in \mathscr{K}$ such that $(U V) \mathscr{L}_{c}(U V)^{*}=\mathscr{L}_{c}$, and finite subsets $\mathscr{P}, \mathscr{Q} \subseteq \mathscr{F}(\mathscr{L})=\mathscr{A}(\mathscr{L})$ such that Ad $U V$ is a bijection of $\mathscr{F}(\mathscr{L}) \backslash \mathscr{P}$ onto $\mathscr{F}(\mathscr{L}) \backslash \mathscr{Q}$ and $(U V)\left(\sum_{A \in \mathscr{P}} A\right)(U V)^{*}=\sum_{B \in \mathscr{Q}} B$. Let $e(k)=\operatorname{card}\{A \in \mathscr{A}(\mathscr{L}): \operatorname{dim} A=k$ and $A \in \mathscr{P}\}$ and $f(k)=$ $\operatorname{card}\{A \in \mathscr{A}(\mathscr{L}): \operatorname{dim} A=k$ and $A \in \mathscr{Q}\}$. Then if $c(k)<\infty$, it follows that $e(k)=f(k)$, and (i) then implies that $e(k)=f(k)$ for all $k$. Thus, there is a partial isometry $Y$ such that $\operatorname{Ad} Y$ is a bijection of $\mathscr{F}(\mathscr{L}) \cap \mathscr{P}$ onto $\mathscr{F}(\mathscr{L}) \cap \mathscr{Q}$. Let $W=Y+U V\left(\sum_{A \in \mathscr{P}} A\right)^{\perp}$. Then Ad $W$ implements an automorphism of $\mathscr{L}^{\prime \prime}$ since $W \mathscr{L}_{c} W^{*}=\mathscr{L}_{c}$ and $W \mathscr{L}_{a} W^{*}=\mathscr{L}_{a}$, and therefore implements an automorphism of $\mathscr{L}^{\prime}$ as well. Also, $W$ is a compact perturbation of $U V$ since $Y$ and $\sum_{A \in \mathscr{P}} A$ are compact, and $U V$ is in turn a compact perturbation of $V$ since $U-I \in \mathscr{K}$.

Suppose instead that (ii) holds, and let $A_{0}$ be the one infinite atom. If $V$ is a unitary such that $V\left(\mathscr{L}^{\prime}+\mathscr{K}\right) V^{*}=\mathscr{L}^{\prime}+\mathscr{K}$, then we again obtain a unitary $U$ with $U-I \in \mathscr{K}$ such that $(U V) \mathscr{L}_{c}(U V)^{*}$, and also there are $\sigma$-finite projections $P, Q \in \mathscr{L}^{\prime}$ such that $(U V) P(U V)^{*}=Q$ 
and $(U V) \mathscr{L}_{a}^{P}(U V)^{*}=\mathscr{L}_{a}^{Q}$. Then $\operatorname{Ad} U V$ is a bijection of $\mathscr{F}(\mathscr{L}) \backslash \mathscr{P}$ onto $\mathscr{F}(\mathscr{L}) \backslash \mathscr{Q}$, where $\mathscr{P}=\{A \in \mathscr{F}(\mathscr{L}): A \leq P\}$ and $\mathscr{Q}=\{A \in$ $\mathscr{F}(\mathscr{L}): A \leq Q\}$. Let $P_{0}=P A_{0}$ and $Q_{0}=Q A_{0}$. Since $c(k)<\infty$ for all $k$, it follows that $e(k)=f(k)$ for all $k$, and therefore $\operatorname{dim} P_{0}=$ $\operatorname{dim} Q_{0}$. Now let $Y$ be a partial isometry such that $\operatorname{Ad} Y$ is a bijection of $\mathscr{F}(\mathscr{L}) \cap \mathscr{P}$ onto $\mathscr{F}(\mathscr{L}) \cap \mathscr{Q}$ and also $Y P_{0} Y^{*}=Q_{0}$. Finally, define $W=Y+U V P^{\perp}$. As in the argument for case (i), $V-W \in \mathscr{K}$ and $W \mathscr{L}^{\prime} W^{*}=\mathscr{L}^{\prime}$.

To prove that either (i) or (ii) is necessary, first suppose that $\mathscr{L} \subseteq$ $\mathscr{B}(\mathscr{H})$ has at least two infinite atoms $A_{0}$ and $A_{1}$. Let $P_{0} \leq A_{0}$ be a rank-one projection and let $F=I-A_{0}-A_{1}$. Define $V$ to be a unitary such that $\left.V\right|_{F \mathscr{H}}=\left.I\right|_{F \mathscr{H}}, V\left(A_{0}-P_{0}\right) V^{*}=A_{0}$, and $V\left(A_{1}+P_{0}\right) V^{*}=$ $A_{1}$. Then certainly $V\left(\mathscr{L}^{\prime}+\mathscr{K}\right) V^{*}=\mathscr{L}^{\prime}+\mathscr{K}$. However, there is no unitary $W$ with $V-W \in \mathscr{K}$ such that $W \mathscr{L}^{\prime} W^{*}=\mathscr{L}^{\prime}$. For if there were, it would follow that $W \mathscr{L}^{\prime \prime} W^{*}=\mathscr{L}^{\prime \prime}$, and therefore $W A_{0} W^{*}$ is an infinite atom of $\mathscr{L}$. But $W A_{0} W^{*}=A_{0}+$ compact, so in fact $W A_{0} W^{*}=A_{0}$. Similarly, $W A_{1} W^{*}=A_{1}$. Let $R=A_{1}+$ $P_{0}$ and $S=A_{1}+W P_{0} W^{*}$. Then, viewing $\left.S W\right|_{R \mathscr{R}}$ and $\left.S V\right|_{R \mathscr{E}}$ as operators from $R \mathscr{H}$ to $S \mathscr{C}$, ind $\left(\left.S W\right|_{R \mathscr{C}}\right)=0$ and ind $\left(\left.S V\right|_{R \mathscr{L}}\right)=$ -1 , contradicting the fact that the Fredholm index is invariant under compact perturbations.

Next, suppose $c(\infty)=1$ and $c\left(k_{0}\right)=\infty$. Let $A_{0}$ be the infinite atom and let $E_{1}, E_{2}, \ldots$ be the atoms of dimension $k_{0}$. Let $G=$ $I-A_{0}-\sum_{i=1}^{\infty} E_{i}$ and define $V$ to be a unitary such that $\left.V\right|_{G \mathscr{K}}=$ $\left.I\right|_{G \mathscr{K}}, V\left(A_{0}+E_{1}\right) V^{*}=A_{0}$, and $V E_{i} V^{*}=E_{i-1}$ for all $i \geq 2$. Then $V\left(\mathscr{L}^{\prime}+\mathscr{K}\right) V^{*}=\mathscr{L}^{\prime}+\mathscr{K}$, but again there is no unitary $W$ with $V-W \in \mathscr{K}$ and $W \mathscr{L}^{\prime} W^{*}=\mathscr{L}^{\prime}$. For $W$ would have to satisfy $W A_{0} W^{*}=A_{0}$, so if $R=A_{0}+E_{1}$ and $S=A_{0}+W E_{1} W^{*}$, then $\operatorname{ind}\left(\left.S W\right|_{R \mathscr{E}}\right)=0$ and $\operatorname{ind}\left(\left.S V\right|_{R \mathscr{K}}\right)=-k_{0}$, a contradiction.

Finally, if $c(\infty)=0, c\left(k_{0}\right)=\infty$, and $c\left(k_{1}\right)=\infty$ for $k_{0} \neq k_{1}$, then a minor variation of the argument given in [P, Corollary 12] yields a unitary which implements an automorphism of $\mathscr{L}^{\prime}+\mathscr{K}$ but does not split.

We can also extend [P, Corollary 13] to the general situation.

THEOREM 12. Let $\mathscr{L}$ be a CSL. Then all automorphisms of $\mathscr{L}^{\prime}+\mathscr{K}$ are inner if and only if

(i) $\mathscr{L}$ is purely atomic,

(ii) $i(\mathscr{L}) \leq 1$, and 
(iii) there is a finite set $\mathscr{S} \subseteq \mathscr{A}(\mathscr{L})$ such that $\operatorname{dim} A \neq \operatorname{dim} B$ for all distinct atoms $A, B \in \mathscr{A}(\mathscr{L}) \backslash \mathscr{S}$.

Proof. Again, by replacing $\mathscr{L}$ with the complemented lattice generated by $\mathscr{L}$, we can assume without loss of generality that $\mathscr{L}$ is complemented. Suppose (i)-(iii) hold, and suppose AdV is an automorphism of $\mathscr{L}^{\prime}+\mathscr{K}$. Then Theorem 11 implies that $V$ splits, since $c(k)<\infty$ for all $k$ by (iii). Thus, $V=W+K$ with $K$ compact and $W$ a unitary satisfying $W \mathscr{L}^{\prime} W^{*}=\mathscr{L}^{\prime}$. Denote the atoms of $\mathscr{L}$ by $\left\{A_{i}\right\}_{i=1}^{M}, M \leq \infty$. Then it follows from (iii) that there is some $N$ such that $(\operatorname{Ad} W)\left(A_{i}\right)=A_{i}$ for all $i>N$ and, if an infinite atom $A_{\text {inf }}$ exists, then $(\operatorname{Ad} W)\left(A_{\text {inf }}\right)=A_{\text {inf }}$. By increasing $N$ if necessary, we can assume $A_{\text {inf }}=A_{k}$ for some $k \leq N$. Now let $P=\sum_{i=1}^{N} A_{i}$ if $i(\mathscr{L})=0$ and $P=\sum_{i=1}^{N} A_{i}-A_{k}$ if $i(\mathscr{L})=1$. Then $W P^{\perp} \in \mathscr{L}^{\prime}$ and $W-W P^{\perp} \in \mathscr{K}$, so $V \in \mathscr{L}^{\prime}+\mathscr{K}$.

To show that (i)-(iii) are necessary, first suppose $\mathscr{L}$ is not purely atomic. Let $P_{a}=\sum_{A \in \mathscr{A}(\mathscr{L})} A$ and $P_{c}=I-P_{a}$. Then $\mathscr{L}_{c}$ is a nonatomic complemented CSL on $P_{c} \mathscr{H}$. From the multiplicity theory of abelian von Neumann algebras, there are orthogonal projections $\left\{P_{m}: 1 \leq m \leq \infty\right\}$ such that for $P_{m} \neq 0,\left(\mathscr{L}_{c} \mid P_{m} \mathscr{L}\right)^{\prime \prime}$ is unitarily equivalent via a unitary $X_{m}$ to the algebra $\mathscr{Z}^{(m)}$ defined after Corollary 10. Choose $n$ so that $P_{n} \neq 0$, and recall that $\mu$ is being used to denote Lebesgue measure. Now if we let $f(x)=x^{2}$, then there is a unitary operator $Y$ such that $Y L^{2}([0, f(t)], \mu)^{(n)}=L^{2}([0, t], \mu)^{(n)}$ for all $t \in[0,1]$ [D1, Corollary 7.16]. Let $P_{t}$ be the projection onto $L^{2}([0, t], \mu)^{(n)}$, so $Y P_{f(t)} Y^{*}=P_{t}$ for all $t$, and $Y$ thus implements an automorphism of $\mathscr{Z}^{(n)}$. But then $P_{1 / 4}^{\perp} Y P_{1 / 4} Y^{*}=P_{1 / 4}^{\perp} P_{1 / 2}$, and it follows that $Y \notin\left\{P_{t}\right\}^{\prime}+\mathscr{K}$, for otherwise the left side would be compact but the right side would not. Thus, $X_{n}^{*} Y X_{n} \notin\left(\mathscr{L}_{c} \mid P_{n} \mathscr{K}\right)^{\prime}+\mathscr{H}$, and therefore $V=P_{a}+\sum_{m \neq n} P_{m}+X_{n}^{*} Y X_{n}$ is a unitary which implements an automorphism of $\mathscr{L}^{\prime}+\mathscr{K}$ that is not inner.

Now suppose $\mathscr{L}$ is purely atomic but that $\mathscr{L}$ has at least two infinite atoms. Again, denote the atoms of $\mathscr{L}$ by $\left\{A_{i}\right\}_{i=1}^{M}, M \leq \infty$. By relabeling if necessary, we can assume $A_{1}$ and $A_{2}$ are infinite. Let $R=\sum_{i=3}^{M} A_{i}$ and define a unitary $V=\left.I\right|_{R \mathscr{E}}+W$, where $W$ is a partial isometry satisfying $W A_{1} W^{*}=A_{2}$ and $W A_{2} W^{*}=A_{1}$. Then $V \mathscr{L}^{\prime} V^{*}=\mathscr{L}^{\prime}$, so Ad $V$ certainly implements an isomorphism of $\mathscr{L}^{\prime}+\mathscr{K}$. However, $V-\delta_{\mathscr{L}}(V)=V R^{\perp}=W \notin \mathscr{K}$, and therefore $V \notin \mathscr{L}^{\prime}+\mathscr{K}$ by [W3, Lemma 5]. 
Finally, suppose (i) and (ii) hold, but (iii) is false. Then there is an infinite sequence of atoms $\left\{B_{i}\right\}$ such that $\operatorname{dim} B_{2 k-1}=\operatorname{dim} B_{2 k}$ for $k=1,2,3, \ldots$. Let $S=\sum_{i=1}^{\infty} B_{i}$ and define a unitary $V=\left.I\right|_{S^{\perp} \mathscr{L}}+$ $W$, where $W$ is a partial isometry satisfying $W B_{2 k-1} W^{*}=B_{2 k}$ and $W B_{2 k} W^{*}=B_{2 k-1}$ for all $k$. Then Ad $V$ implements an isomorphism of $\mathscr{L}^{\prime}$, and therefore $\mathscr{L}^{\prime}+\mathscr{K}$ also, but again $V \notin \mathscr{L}^{\prime}+\mathscr{K}$ since $V-\delta_{\mathscr{L}}(V)=V S=W \notin \mathscr{K}$.

The last proof used several techniques for constructing automorphisms which are not inner. These techniques give an indication of how to calculate the outer automorphism group of $\mathscr{L}^{\prime}+\mathscr{K}$. First, we will use $\operatorname{Sym}(S)$ to denote the group of all permutations of a set $S$. Now let $\mathscr{L}$ be a CSL and let $P_{\mathscr{F}}=\sum_{A \in \mathscr{F}(\mathscr{L})} A$. If $E$ is a finite sum of atoms in $\mathscr{F}(\mathscr{L})$, define $\mathscr{L}_{E}$ to be the lattice $\mathscr{L} P_{\mathscr{F}} E^{\perp}$ on $P_{\mathscr{F}} E^{\perp} \mathscr{H}$. Given $\mathscr{L}_{E}$ and $\mathscr{L}_{F}$, let $\operatorname{ISO}\left(\mathscr{L}_{E}, \mathscr{L}_{F}\right)$ denote the set of bijections $\theta: \mathscr{A}\left(\mathscr{L}_{E}\right) \rightarrow \mathscr{A}\left(\mathscr{L}_{F}\right)$ which preserve dimension. Note that $\theta$ extends to a map which takes sums of atoms to sums of atoms. Let $\operatorname{ISO}_{\mathscr{F}}(\mathscr{L})=\bigcup \operatorname{ISO}\left(\mathscr{L}_{E}, \mathscr{L}_{F}\right)$, where the union is taken over all pairs $(E, F)$ of finite sums of atoms in $\mathscr{F}(\mathscr{L})$ with the property that if $i(\mathscr{L})=0$, then $\operatorname{dim} E=\operatorname{dim} F$. Now if $\theta \in$ $\operatorname{ISO}\left(\mathscr{L}_{E}, \mathscr{L}_{F}\right)$ and $G$ is a finite sum of atoms in $\mathscr{L}_{E}$, then define $\theta_{G} \in \mathrm{ISO}\left(\left(\mathscr{L}_{E}\right)_{G},\left(\mathscr{L}_{F}\right)_{\theta(G)}\right)$ by restriction. We can now define an equivalence relation on $\operatorname{ISO}_{\mathscr{F}}(\mathscr{L})$ by $\theta \sim \tau$ if there are finite sums $G$ and $H$ of atoms such that $\theta_{G}=\tau_{H}$. The class of $\theta$ will be denoted by $[\theta]$.

We next define a multiplication on $\operatorname{ISO}_{\mathscr{F}}(\mathscr{L}) / \sim$. Suppose $\theta \in$ $\operatorname{ISO}\left(\mathscr{L}_{E}, \mathscr{L}_{F}\right)$ and $\tau \in \operatorname{ISO}\left(\mathscr{L}_{G}, \mathscr{L}_{H}\right)$, and define $[\tau][\theta]=[\rho]$, where $\rho=\tau_{F G^{\perp}} \circ \theta_{\theta^{-1}\left(F^{\perp} G\right)}$. It is not hard to see that this multiplication is well-defined and associative, [id] is an identity, and $[\theta]^{-1}=\left[\theta^{-1}\right]$. Thus, $\operatorname{ISO}_{\mathscr{F}}(\mathscr{L}) / \sim$ is a group, denoted $a$-ISO $\mathscr{F}(\mathscr{L})$. We note that this group is very similar to, and was motivated by, the group $a$ - Aut $\mathscr{M}$ in [DW, p. 615-616].

We are now in a position to calculate $\operatorname{Out}\left(\mathscr{L}^{\prime}+\mathscr{K}\right)$. For simplicity, we will compute this group separately for the purely atomic and nonatomic cases, and then combine these results to prove the general case in Corollary 20. In the following theorem, we note that $\operatorname{Sym}(\mathscr{I}(\mathscr{L}))=\{$ id $\}$ if $i(\mathscr{L})=0$ or 1 , and $a-\operatorname{ISO}_{\mathscr{F}}(\mathscr{L})=\{[$ id] $\}$ if $\mathscr{F}(\mathscr{L})$ is finite.

Theorem 13. Suppose $\mathscr{L}$ is a purely atomic CSL. Then

$$
\operatorname{Out}\left(\mathscr{L}^{\prime}+\mathscr{K}\right) \cong \operatorname{Sym}(\mathscr{I}(\mathscr{L})) \times a-I S O_{\mathscr{F}}(\mathscr{L}) .
$$


The proof will be completed by a succession of lemmas. First, note that each of the groups in the statement of the theorem is the same for $\mathscr{L}$ as for the complemented lattice generated by $\mathscr{L}$, so we can assume $\mathscr{L}$ is complemented. After the next technical lemma, we will define a map $\Gamma: \operatorname{Aut}\left(\mathscr{L}^{\prime}+\mathscr{K}\right) \rightarrow \operatorname{Sym}(\mathscr{I}(\mathscr{L})) \times a-\operatorname{ISO}_{\mathscr{F}}(\mathscr{L})$, and then show that $\Gamma$ is a surjective group homomorphism whose kernel is $\operatorname{Inn}\left(\mathscr{L}^{\prime}+\mathscr{K}\right)$, completing the proof.

LEMMA 14. Suppose $\mathscr{L}$ is a complemented CSL and $W$ is a unitary in $\mathscr{L}^{\prime}+\mathscr{K}$ such that Ad $W$ is a bijection of $\mathscr{F}(\mathscr{L}) \backslash \mathscr{R}$ onto $\mathscr{F}(\mathscr{L}) \backslash \mathscr{S}$ for some finite sets $\mathscr{R}, \mathscr{S} \subseteq \mathscr{F}(\mathscr{L})$. Then there is a finite set $\widetilde{\mathscr{R}} \supseteq \mathscr{R}$ such that $(\operatorname{Ad} W)(A)=A$ for all $A \in \mathscr{F}(\mathscr{L}) \backslash \widetilde{R}$.

Proof. Write $W=S+K$ for some $S \in \mathscr{L}^{\prime}$ and $K \in \mathscr{K}$, and suppose $(\operatorname{Ad} W)\left(E_{i}\right) \neq E_{i}$ for an infinite set $\left\{E_{i}: 1 \leq i<\infty\right\} \subseteq$ $\mathscr{F}(\mathscr{L}) \backslash \mathscr{R}$. Let $e_{i} \in E_{i} \mathscr{H}$ with $\left\|e_{i}\right\|=1$, and let $f_{i}=W e_{i} .\left\|K e_{i}\right\| \rightarrow$ 0 since $e_{i} \rightarrow 0$ weakly and $K$ is compact. But then

$$
\begin{aligned}
1 & =\left\langle f_{i}, f_{i}\right\rangle=\left\langle E_{i}^{\perp} W E_{i} e_{i}, f_{i}\right\rangle=\left\langle E_{i}^{\perp}(S+K) E_{i} e_{i}, f_{i}\right\rangle \\
& =\left\langle E_{i}^{\perp} K E_{i} e_{i}, f_{i}\right\rangle=\left\langle K e_{i}, E_{i}^{\perp} f_{i}\right\rangle,
\end{aligned}
$$

a contradiction.

If $\alpha \in \operatorname{Aut}\left(\mathscr{L}^{\prime}+\mathscr{K}\right)$, then $\alpha=\operatorname{Ad} V$ for some unitary $V$ by Lemma 2. If $\operatorname{Ad} V=\operatorname{Ad} V^{\prime}$ on $\mathscr{L}^{\prime}+\mathscr{K}$, then $V^{*} V^{\prime} \in\left(\mathscr{L}^{\prime}+\mathscr{K}\right)^{\prime}=\mathbb{C} I$, so $V$ is unique up to multiplication by a scalar of modulus 1 . By Lemma 8 , there is a unitary $U$ with $U-I \in \mathscr{K}$ and $\sigma$-finite projections $P, Q \in \mathscr{L}^{\prime}$ such that $(\operatorname{Ad} U V)(P)=Q$ and $(\operatorname{Ad} U V)\left(\mathscr{L}^{P}\right)=\mathscr{L}^{Q}$. Thus, Ad $U V$ is a bijection of $\mathscr{I}\left(\mathscr{L}^{P}\right)$ onto $\mathscr{I}\left(\mathscr{L}^{Q}\right)$. Because of the form of $P$ and $Q$, this induces a unique bijection $l_{\alpha}$ of $\mathscr{I}(\mathscr{L})$ satisfying $l_{\alpha}(A) Q^{\perp}=(\operatorname{Ad} U V)\left(A P^{\perp}\right)$ for all $A \in \mathscr{I}(\mathscr{L})$. It follows that $\imath_{\alpha}(A)-\alpha(A) \in \mathscr{K}$ for all $A \in \mathscr{I}(\mathscr{L})$. From the above, a different choice of $V$ has no effect on $\operatorname{Ad} U V$. Suppose $U^{\prime}, P^{\prime}$, and $Q^{\prime}$ satisfy the same properties as $U, P$, and $Q$, and $l_{\alpha}^{\prime}$ is the corresponding induced bijection of $\mathscr{I}(\mathscr{L})$. But then $l_{\alpha}(A)-l_{\alpha}^{\prime}(A) \in \mathscr{K}$ for all $A \in \mathscr{I}(\mathscr{L})$, so $l_{\alpha}=l_{\alpha}^{\prime}$. This shows that $l_{\alpha}$ is well-defined, i.e., it is determined by any $V$ implementing $\alpha$ and any $U, P$, and $Q$ satisfying Lemma 8 for $V$ and $\mathscr{L}$. An equivalent characterization of $l_{\alpha}$ is that it is the unique bijection of $\mathscr{I}(\mathscr{L})$ such that $l_{\alpha}(A)-\alpha(A) \in$ $\mathscr{K}$ for all $A \in \mathscr{I}(\mathscr{L})$.

Now Ad $U V$ is also a bijection of $\mathscr{F}(\mathscr{L}) \backslash \mathscr{P}$ onto $\mathscr{F}(\mathscr{L}) \backslash \mathscr{Q}$, where $\mathscr{P}=\{A \in \mathscr{F}(\mathscr{L}): A \leq P\}$ and $\mathscr{Q}=\{A \in \mathscr{F}(\mathscr{L}): A \leq Q\}$. 
Thus, $\left.(\operatorname{Ad} U V)\right|_{\mathscr{F}(\mathscr{L}) \backslash \mathscr{P}} \in \operatorname{ISO}\left(\mathscr{L}_{E}, \mathscr{L}_{F}\right)$, where $E=\sum_{A \in \mathscr{P}} A$ and $F=\sum_{A \in \mathscr{Q}} A$. Moreover, if $i(\mathscr{L})=0$, then $E=P$ and $F=Q$, so $\operatorname{dim} E=\operatorname{dim} F$. Let $\varphi_{\alpha}=\left[\left.(\operatorname{Ad} U V)\right|_{\mathscr{F}(\mathscr{L}) \backslash \mathscr{P}}\right]$. If $U^{\prime}, P^{\prime}$, and $Q^{\prime}$ satisfy the same properties as $U, P$, and $Q$, we will obtain analogous sets $\mathscr{P}^{\prime}$ and $\mathscr{Q}^{\prime}$ and projections $E^{\prime}$ and $F^{\prime}$. Let

$$
\mathscr{R}=\mathscr{P} \cup\left\{\left(\operatorname{Ad} V^{*} U^{*}\right)(A): A \in \mathscr{Q}^{\prime} \backslash \mathscr{Q}\right\}
$$

and

$$
\mathscr{S}=\mathscr{P}^{\prime} \cup\left\{\left(\operatorname{Ad} V^{*} U^{\prime *}\right)(A): A \in \mathscr{Q} \backslash \mathscr{Q}^{\prime}\right\} .
$$

Then $\operatorname{Ad} V^{*} U^{\prime *} U V$ is a bijection of $\mathscr{F}(\mathscr{L}) \backslash \mathscr{R}$ onto $\mathscr{F}(\mathscr{L}) \backslash \mathscr{S}$ and $V^{*} U^{*} U V=I+$ compact, so Lemma 14 , with $W=V^{*} U^{* *} U V$, implies that there is a finite set $\widetilde{R} \supseteq \mathscr{R}$ such that $(\operatorname{Ad} U V)(A)=$ $\left(\right.$ Ad $\left.U^{\prime} V\right)(A)$ for all $A \in \mathscr{F}(\mathscr{L}) \backslash \widetilde{\mathscr{R}}$. Therefore $\left.(\operatorname{Ad} U V)\right|_{\mathscr{F}(\mathscr{L}) \backslash \mathscr{P}} \sim$ (Ad $\left.U^{\prime} V\right)\left.\right|_{\mathscr{F}(\mathscr{L}) \backslash \mathscr{P}^{\prime}}$, and it follows that $\varphi_{\alpha}$ is well-defined. Thus, we have proved

LEMMA 15. The map $\Gamma: \operatorname{Aut}\left(\mathscr{L}^{\prime}+\mathscr{K}\right) \rightarrow \operatorname{Sym}(\mathscr{I}(\mathscr{L})) \times a-I S O_{\mathscr{F}}(\mathscr{L})$ by $\Gamma(\alpha)=\left(l_{\alpha}, \varphi_{\alpha}\right)$ is well-defined.

LEMMA 16. $\Gamma$ is surjective.

Proof. Suppose $(l,[\varphi]) \in \operatorname{Sym}(\mathscr{I}(\mathscr{L})) \times a$-ISO $\mathscr{F}(\mathscr{L})$. Let $\psi \in[\varphi]$, so $\psi \in \operatorname{ISO}\left(\mathscr{L}_{E}, \mathscr{L}_{F}\right)$ for some $E, F$. Let $W$ be a partial isometry such that $W A W^{*}=\psi(A)$ for all $A \in \mathscr{A}\left(\mathscr{L}_{E}\right)=\mathscr{F}\left(\mathscr{L}_{E}\right)$ and $W A W^{*}=\imath(A)$ for all $A \in \mathscr{I}(\mathscr{L})$. If $\operatorname{dim} E=\operatorname{dim} F$ (in particular, if $i(\mathscr{L})=0)$, let $X$ be a partial isometry satisfying $X E X^{*}=F$, and define $V=X+W, P_{0}=0$, and $Q_{0}=0$. On the other hand, if $\operatorname{dim} E \neq \operatorname{dim} F$ (so $i(\mathscr{L})>0$ ), choose an infinite atom $A_{0}$ and finite subprojections $P_{0}<A_{0}$ and $Q_{0}<l\left(A_{0}\right)$ such that $\operatorname{dim}\left(E+P_{0}\right)=\operatorname{dim}\left(F+Q_{0}\right)$. Let $Y$ be a partial isometry with $Y\left(A_{0} P_{0}^{\perp}\right) Y^{*}=l\left(A_{0}\right) Q_{0}^{\perp}$ and $Y\left(E+P_{0}\right) Y^{*}=F+Q_{0}$, and define $V=$ $Y+W\left(E+A_{0}\right)^{\perp}$. Then in either case $(\operatorname{Ad} V)\left(\mathscr{L}^{E+P_{0}}\right)=\mathscr{L}^{F+Q_{0}}$, so $\alpha=\operatorname{Ad} V$ is an automorphism of $\mathscr{L}^{\prime}+\mathscr{K}$, and it is readily apparent that $\Gamma(\alpha)=(l,[\varphi])$ (simply let $U=I, P=E+P_{0}$, and $\left.Q=F+Q_{0}\right)$.

LEMMA 17. $\Gamma$ is a group homomorphism.

Proof. Let $\alpha_{1}, \alpha_{2} \in \operatorname{Aut}\left(\mathscr{L}^{\prime}+\mathscr{K}\right)$ with $\alpha_{1}=\operatorname{Ad} V_{1}$ and $\alpha_{2}=$ Ad $V_{2}$. Define $\alpha_{3}=\alpha_{2} \circ \alpha_{1}=\operatorname{Ad} V_{2} V_{1}$. Use Lemma 8 to obtain unitaries $U_{1}, U_{2}$, and $U_{3}$ with $U_{i}-I \in \mathscr{K}$ and $\sigma$-finite projections 
$P_{1}, P_{2}, P_{3}, Q_{1}, Q_{2}, Q_{3}$ in $\mathscr{L}^{\prime}$ such that $\left(\operatorname{Ad} U_{i} V_{i}\right)\left(P_{i}\right)=Q_{i}$ and $\left(\operatorname{Ad} U_{i} V_{i}\right)\left(\mathscr{L}^{P_{i}}\right)=\mathscr{L}^{Q_{i}}$ for $i=1,2,\left(\operatorname{Ad} U_{3} V_{2} V_{1}\right)\left(P_{3}\right)=Q_{3}$, and $\left(\operatorname{Ad} U_{3} V_{2} V_{1}\right)\left(\mathscr{L}^{P_{3}}\right)=\mathscr{L}^{Q_{3}}$. Then $l_{\alpha_{i}}(A)-\alpha_{i}(A) \in \mathscr{K}$ for all $A \in$ $\mathscr{I}(\mathscr{L})$, so $l_{\alpha_{2}}\left(l_{\alpha_{1}}(A)\right)-\alpha_{2}\left(l_{\alpha_{1}}(A)\right) \in \mathscr{K}$. But then

$$
\begin{aligned}
\left(l_{\alpha_{2}} \circ l_{\alpha_{1}}\right)(A)-\alpha_{3}(A)= & l_{\alpha_{2}}\left(l_{\alpha_{1}}(A)\right)-\alpha_{2}\left(l_{\alpha_{1}}(A)\right) \\
& +\alpha_{2}\left(l_{\alpha_{1}}(A)\right)-\alpha_{2}\left(\alpha_{1}(A)\right) \\
= & l_{\alpha_{2}}\left(l_{\alpha_{1}}(A)\right)-\alpha_{2}\left(l_{\alpha_{1}}(A)\right) \\
& +V_{2}\left(l_{\alpha_{1}}(A)-\alpha_{1}(A)\right) V_{2}^{*} \in \mathscr{K},
\end{aligned}
$$

and it 1ollows that $l_{\alpha_{3}}=l_{\alpha_{2}} \circ l_{\alpha_{1}}$.

Now let $\mathscr{P}_{i}=\left\{A \in \mathscr{F}(\mathscr{L}): A \leq P_{i}\right\}$ and $\mathscr{Q}_{i}=\{A \in \mathscr{F}(\mathscr{L}): A \leq$ $\left.Q_{i}\right\}, i=1,2,3$, so $\varphi_{\alpha_{t}}=\left[\left.\left(\operatorname{Ad} U_{i} V_{i}\right)\right|_{\mathscr{F}(\mathscr{L}) \mid \mathscr{P}_{i}}\right]$ for $i=1,2$, and $\varphi_{\alpha_{3}}=\left[\left.\left(\operatorname{Ad} U_{3} V_{2} V_{1}\right)\right|_{\mathscr{F}(\mathscr{L}) \mid \mathscr{P}_{3}}\right]$. If we define

$$
\mathscr{P}_{4}=\mathscr{P}_{1} \cup\left\{\left(\operatorname{Ad} V_{1}^{*} U_{1}^{*}\right)(A): A \in \mathscr{P}_{2} \backslash \mathscr{Q}_{1}\right\}
$$

and

$$
\mathscr{Q}_{4}=\mathscr{Q}_{2} \cup\left\{\left(\operatorname{Ad} U_{2} V_{2}\right)(A): A \in Q_{1} \backslash P_{2}\right\},
$$

then $\operatorname{Ad} U_{2} V_{2} U_{1} V_{1}$ is a bijection of $\mathscr{F}(\mathscr{L}) \backslash \mathscr{P}_{4}$ onto $\mathscr{F}(\mathscr{L}) \backslash \mathscr{Q}_{4}$, and $\varphi_{\alpha_{2}} \varphi_{\alpha_{1}}=\left[\left.\left(\operatorname{Ad} U_{2} V_{2} U_{1} V_{1}\right)\right|_{\mathscr{F}(\mathscr{L})(\mathscr{P}}\right]$ by definition of the product in $a$-ISO $F / \sim$. Define $X=U_{3} V_{2} V_{1}$ and $Y=U_{2} V_{2} U_{1} V_{1}$, and let

$$
\mathscr{R}=\mathscr{P}_{3} \cup\left\{\left(\operatorname{Ad} X^{*}\right)(A): A \in \mathscr{Q}_{4} \backslash \mathscr{Q}_{3}\right\}
$$

and

$$
\mathscr{S}=\mathscr{P}_{4} \cup\left\{\left(\operatorname{Ad} Y^{*}\right)(A): A \in \mathscr{Q}_{3} \backslash \mathscr{Q}_{4}\right\} .
$$

Then $\operatorname{Ad}^{*} X$ is a bijection of $\mathscr{F}(\mathscr{L}) \backslash \mathscr{R}$ onto $\mathscr{F}(\mathscr{L}) \backslash \mathscr{S}$ and $Y^{*} X=I+$ compact, so Lemma 14 , with $W=Y^{*} X$, implies that $(\operatorname{Ad} X)(A)=(\operatorname{Ad} Y)(A)$ for all $A \in \mathscr{F}(\mathscr{L}) \backslash \widetilde{\mathscr{R}}$, where $\widetilde{\mathscr{R}}$ is some finite set containing $\mathscr{R}$. Therefore $\left.\left.(\operatorname{Ad} X)\right|_{\mathscr{F}(\mathscr{L}) \backslash \mathscr{P}_{3}} \sim(\operatorname{Ad} Y)\right|_{\mathscr{F}(\mathscr{L}) \mid \mathscr{P}_{4}}$, i.e., $\varphi_{\alpha_{3}}=\varphi_{\alpha_{2}} \varphi_{\alpha_{1}}$.

LEMMA 18. $\operatorname{Ker}(\Gamma)=\operatorname{Inn}\left(\mathscr{L}^{\prime}+\mathscr{K}\right)$.

Proof. First suppose $\theta \in \operatorname{Inn}\left(\mathscr{L}^{\prime}+\mathscr{K}\right), \theta=\operatorname{Ad} V$. Let $U$ be any unitary and $P, Q \in \mathscr{L}^{\prime}$ be any $\sigma$-finite projections satisfying Lemma 8. Suppose $\imath_{\theta}(A) \neq A$ for some $A \in \mathscr{I}(\mathscr{L})$. Write $V=S+K$ with $S \in \mathscr{L}^{\prime}$ and $K \in \mathscr{K}$. Then $A^{\perp} V A V^{*}=A^{\perp}(S+K) A V^{*}=$ $A^{\perp} K A V^{*} \in \mathscr{K}$. Also, $l_{\theta}(A)-V A V^{*}=l_{\theta}(A)-\theta(A) \in \mathscr{K}$. It follows that $l_{\theta}(A)=A^{\perp}\left(l_{\theta}(A)-A\right)=A^{\perp}\left(l_{\theta}(A)-V A V^{*}\right)+A^{\perp}\left(V A V^{*}-A\right) \epsilon$ $\mathscr{K}$, a contradiction. Therefore, $\imath_{\theta}=\mathrm{id} \in \operatorname{Sym}(\mathcal{I}(\mathscr{L}))$. 
Now Ad $U V$ is a bijection of $\mathscr{F}(\mathscr{L}) \backslash \mathscr{P}$ onto $\mathscr{F}(\mathscr{L}) \backslash \mathscr{Q}$ for some finite sets $\mathscr{P}, \mathscr{Q} \subseteq \mathscr{F}(\mathscr{L}) . U V \in \mathscr{L}^{\prime}+\mathscr{K}$ since $U-I \in \mathscr{K}$, so Lemma 14 implies that there is a finite set $\widetilde{P} \supseteq \mathscr{P}$ such that $(\operatorname{Ad} U V)(A)=A$ for all $A \in \mathscr{F}(\mathscr{L}) \backslash \widetilde{\mathscr{P}}$. It follows that $\varphi_{\theta}=$ [id], and therefore $\Gamma(\theta)=($ id,$[$ id] $)$.

Suppose on the other hand that $\Gamma(\theta)=(\mathrm{id},[\mathrm{id}])$. We want to show that $\theta=\operatorname{Ad} V \in \operatorname{Inn}\left(\mathscr{L}^{\prime}+\mathscr{K}\right)$. Let $U$ be any unitary and $P, Q \in \mathscr{L}^{\prime}$ be any $\sigma$-finite projections satisfying Lemma 8. Let $\mathscr{P}=$ $\{A \in \mathscr{F}(\mathscr{L}): A \leq P\}$ and $\mathscr{Q}=\{A \in \mathscr{F}(\mathscr{L}): A \leq Q\}$. Then Ad $U V$ is a bijection of $\mathscr{F}(\mathscr{L}) \backslash \mathscr{P}$ onto $\mathscr{F}(\mathscr{L}) \backslash \mathscr{Q}$, and $\left.($ Ad $U V)\right|_{\mathscr{F}(\mathscr{L}) \backslash \mathscr{P}} \sim$ id by the definition of $\varphi_{\theta}$. It follows that there is a finite set $\widetilde{\mathscr{P}} \supseteq \mathscr{P}$ such that $\left.(\operatorname{Ad} U V)\right|_{\mathscr{F}(\mathscr{L}) \backslash \tilde{\mathscr{P}}}=\left.\mathrm{id}\right|_{\mathscr{F}(\mathscr{L}) \backslash \widetilde{\mathscr{P}}}$. Also, Ad $U V$ is a bijection of $\mathscr{I}\left(\mathscr{L}^{P}\right)$ onto $\mathscr{I}\left(\mathscr{L}^{Q}\right)$ such that $A Q^{\perp}=(\operatorname{Ad} U V)\left(A P^{\perp}\right)$ for all $A \in \mathscr{I}(\mathscr{L})$. Let $\mathscr{R}$ be the finite set $\widetilde{\mathscr{P}} \cup\{A \in \mathscr{I}(\mathscr{L}): A P \neq 0$ or $A Q \neq 0\}$, and define $\widetilde{P}=\sum_{A \in \widetilde{P}} A$ and $R=\sum_{A \in \mathscr{R}} A$. Then for $T \in \mathscr{L}^{\prime \prime}$,

$$
\begin{aligned}
U V T V^{*} U^{*}= & U V T R^{\perp} V^{*} U^{*}+U V T R V^{*} U^{*} \\
= & T R^{\perp}+U V T R \widetilde{P}^{\perp} P^{\perp} V^{*} U^{*}+U V T R \widetilde{P} P^{\perp} V^{*} U^{*} \\
& +U V T R P V^{*} U^{*} \\
= & T R^{\perp}+T R \widetilde{P}^{\perp} Q^{\perp}+U V T \widetilde{P} P^{\perp} V^{*} U^{*}+U V T P V^{*} U^{*} \\
= & T+\text { compact. }
\end{aligned}
$$

Equivalently, $(U V) T-T(U V) \in \mathscr{K}$ for all $T \in \mathscr{L}^{\prime \prime}$. Therefore, $U V \in \mathscr{L}^{\prime}+\mathscr{K}$ by [JP, Theorem 2.1], so $V \in \mathscr{L}^{\prime}+\mathscr{K}$ also since $U-I \in \mathscr{K}$.

Proof of Theorem 13. Apply Lemmas 15-18.

THEOREM 19. If $\mathscr{L}$ is a nonatomic CSL, then

$$
\operatorname{Out}\left(\mathscr{L}^{\prime}+\mathscr{K}\right) \cong s-\operatorname{Aut}\left(\mathscr{L}^{\prime \prime}\right) \text {. }
$$

Proof. As before, we can assume $\mathscr{L}$ is complemented. Let $\alpha=$ Ad $V \in \operatorname{Aut}\left(\mathscr{L}^{\prime}+\mathscr{K}\right)$, and let $U, P$, and $Q$ be given by Lemma 8 . Then $P=Q=0$ by Lemma 1 , so $(\operatorname{Ad} U V)(\mathscr{L})=\mathscr{L}$, and therefore Ad $U V$ also implements an automorphism of $\mathscr{L}^{\prime \prime}$. By the same reasoning used in the proof of Theorem 13, another choice for $V$ has no effect on $\operatorname{Ad} U V$. If $U^{\prime}, P^{\prime}$, and $Q^{\prime}$ satisfy the same properties as $U, P$, and $Q$, then $(\operatorname{Ad} U V)(E)-\left(\operatorname{Ad} U^{\prime} V\right)(E) \in \mathscr{K}$ for every projection $E \in \mathscr{L}$, and this implies that $(\operatorname{Ad} U V)(E)=\left(\operatorname{Ad} U^{\prime} V\right)(E)$ 
since $E$ is infinite. Thus, the map $\gamma: \operatorname{Aut}\left(\mathscr{L}^{\prime}+\mathscr{K}\right) \rightarrow s-\operatorname{Aut}\left(\mathscr{L}^{\prime \prime}\right)$ by $\gamma(\alpha)=\operatorname{Ad} U V$ is well-defined, i.e., every possible choice of $V, U$, $P$, and $Q$ yields the same automorphism of $\mathscr{L}^{\prime \prime}$.

Now if $\operatorname{Ad} W \in s-\operatorname{Aut}\left(\mathscr{L}^{\prime \prime}\right)$, then $\operatorname{Ad} W \in \operatorname{Aut}\left(\mathscr{L}^{\prime}+\mathscr{K}\right)$ also. $U=I$ and $P=Q=0$ satisfy Lemma 8 , so $\gamma(\operatorname{Ad} W)=\operatorname{Ad} W$. This shows that $\gamma$ is surjective.

Suppose $\alpha, \beta \in \operatorname{Aut}\left(\mathscr{L}^{\prime}+\mathscr{H}\right)$ with $\alpha=\operatorname{Ad} V_{1}$ and $\beta=\operatorname{Ad} V_{2}$. Let $U_{1}, U_{2}$, and $U_{3}$ be unitaries with $U_{i}-I \in \mathscr{K}$ such that $\operatorname{Ad} U_{1} V_{1}$, $\operatorname{Ad} U_{2} V_{2}, \operatorname{Ad} U_{3} V_{2} V_{1} \in \operatorname{Aut}\left(\mathscr{L}^{\prime \prime}\right)$, i.e., $\gamma(\alpha)=\operatorname{Ad} U_{1} V_{1}, \gamma(\beta)=$ $\operatorname{Ad} U_{2} V_{2}$, and $\gamma(\beta \circ \alpha)=\operatorname{Ad} U_{3} V_{2} V_{1}$. But then

$$
\left(\operatorname{Ad} U_{2} V_{2} \circ \operatorname{Ad} U_{1} V_{1}\right)(E)-\left(\operatorname{Ad} U_{3} V_{2} V_{1}\right)(E) \in \mathscr{K}
$$

for every projection $E \in \mathscr{L}$, and therefore

$$
\left(\operatorname{Ad} U_{2} V_{2} \circ \operatorname{Ad} U_{1} V_{1}\right)(E)=\left(\operatorname{Ad} U_{3} V_{2} V_{1}\right)(E) .
$$

Thus $\gamma(\beta) \circ \gamma(\alpha)=\gamma(\beta \circ \alpha)$, and $\gamma$ is a group homomorphism.

Now suppose $\alpha=\operatorname{Ad} V \in \operatorname{Inn}\left(\mathscr{L}^{\prime}+\mathscr{K}\right)$. Let $\gamma(\alpha)=\operatorname{Ad} U V$, and write $U=I+K, V=S+L$ with $K, L \in \mathscr{K}$ and $S \in \mathscr{L}^{\prime}$. Then for each projection $E \in \mathscr{L},(\operatorname{Ad} U V)(E)=(I+K)(S+L) E V^{*} U^{*}=$ $E S V^{*} U^{*}+M$ for some $M \in \mathscr{K}$. Therefore, $E^{\perp}(\operatorname{Ad} U V)(E) \in \mathscr{H}$, a contradiction unless $E^{\perp}(\operatorname{Ad} U V)(E)=0$. But the same argument applied to $E^{\perp}$ shows that $E(\operatorname{Ad} U V)\left(E^{\perp}\right)=0$, and these two identities imply that $(\operatorname{Ad} U V)(E)=E$ for all $E \in \mathscr{L}$. Therefore, $\gamma(\alpha)=$ id.

Finally, suppose $\alpha=\operatorname{Ad} V \in \operatorname{Aut}\left(\mathscr{L}^{\prime}+\mathscr{K}\right)$ and $\gamma(\alpha)=\operatorname{Ad} U V=$ id $\in s$-Aut $\left(\mathscr{L}^{\prime \prime}\right)$. Then $U V \in \mathscr{L}^{\prime}$, so $V \in \mathscr{L}^{\prime}+\mathscr{K}$, i.e., $\alpha \in$ $\operatorname{Inn}\left(\mathscr{L}^{\prime}+\mathscr{K}\right)$. Therefore, $\operatorname{Ker}(\gamma)=\operatorname{Inn}\left(\mathscr{L}^{\prime}+\mathscr{K}\right)$, and the proof is complete.

Corollary 20. If $\mathscr{L}$ is a CSL, then

$$
\operatorname{Out}\left(\mathscr{L}^{\prime}+\mathscr{K}\right) \cong s-\operatorname{Aut}\left(\mathscr{L}_{c}^{\prime \prime}\right) \times \operatorname{Sym}(\mathscr{I}(\mathscr{L})) \times a-I S O_{\mathscr{F}}(\mathscr{L}) .
$$

Proof. Again, we can assume without loss of generality that $\mathscr{L}$ is complemented. Let $\alpha=\operatorname{Ad} V \in \operatorname{Aut}\left(\mathscr{L}^{\prime}+\mathscr{K}\right)$, and let $U, P$, and $Q$ be given by Lemma 8. Let $P_{a}=\sum_{A \in \mathscr{A}(\mathscr{L})} A$ and $P_{c}=I-P_{a}$. Then $(\operatorname{Ad} U V)\left(\mathscr{L}_{c}\right)=\mathscr{L}_{c}$ and $(\operatorname{Ad} U V)\left(\mathscr{L}_{a}^{P}\right)=\mathscr{L}_{a}^{Q}$, so $\left.(\operatorname{Ad} U V)\right|_{P_{c} \mathscr{L}}$ implements an automorphism $\alpha_{c}$ of $\mathscr{L}_{c}^{\prime}+\mathscr{H}\left(P_{c} \mathscr{H}\right)$ and (Ad $\left.U V\right)\left.\right|_{P_{a} \mathscr{H}}$ implements an automorphism $\alpha_{a}$ of $\mathscr{L}_{a}^{\prime}+\mathscr{K}\left(P_{a} \mathscr{H}\right)$. In addition, $\left(\alpha_{c} \oplus \alpha_{a}\right) \circ \alpha^{-1} \in \operatorname{Inn}\left(\mathscr{L}^{\prime}+\mathscr{K}\right)$ since $U-I \in \mathscr{K}$. Thus, by defining 
$\widetilde{\Gamma}(\alpha)=\left(\gamma\left(\alpha_{c}\right), \Gamma\left(\alpha_{a}\right)\right)=\left(\gamma\left(\alpha_{c}\right), l_{\alpha_{a}}, \varphi_{\alpha_{a}}\right)$, it follows from Theorems 13 and 19 that $\widetilde{\Gamma}$ induces the desired isomorphism.

Since any unitary which implements an automorphism of $\mathscr{L}_{c}^{\prime \prime}$ must map $\mathscr{C}\left(\mathscr{L}_{c}\right)$ onto itself, Corollary 20 thus gives a characterization of $\operatorname{Out}\left(\mathscr{L}^{\prime}+\mathscr{K}\right)$ in terms of certain maps on $\mathscr{C}(\mathscr{L})$. Of course, $\operatorname{Sym}(\mathscr{I}(\mathscr{L}))$ is isomorphic to the symmetric group on $n$ letters if $i(\mathscr{L})=n$, and $\operatorname{Sym}(\mathscr{I}(\mathscr{L}))=\operatorname{Sym}(\mathbb{N})$ if $i(\mathscr{L})=\infty$. In addition, $\operatorname{Out}\left(\mathscr{L}_{c}^{\prime}\right)$ provides an alternate description of $s$-Aut $\left(\mathscr{L}_{c}^{\prime \prime}\right)$. To see this, first note that every element of $s-\operatorname{Aut}\left(\mathscr{L}_{c}^{\prime \prime}\right)$ extends to an automorphism of $\mathscr{L}_{c}^{\prime}$. On the other hand, every automorphism of $\mathscr{L}_{c}^{\prime}$ is spatial by [Di, §III.3.2, Corollary 1], and thus defines an element of $s$-Aut $\left(\mathscr{L}_{c}^{\prime \prime}\right)$ by restriction. Moreover, if $\operatorname{Ad} U \in \operatorname{Aut}\left(\mathscr{L}_{c}^{\prime}\right)$, then Ad $U=$ id on $\mathscr{L}_{c}^{\prime \prime}$ if and only if $U \in \mathscr{L}_{c}^{\prime}$, i.e., $\operatorname{Ad} U \in \operatorname{Inn}\left(\mathscr{L}_{c}^{\prime}\right)$. It follows that $\operatorname{Out}\left(\mathscr{L}^{\prime}+\mathscr{K}\right)$ and $\operatorname{Out}\left(\mathscr{L}^{\prime}\right)$ differ only in the factor $a$-ISO $\operatorname{IF}_{\mathscr{F}}(\mathscr{L}) . \quad s-\operatorname{Aut}\left(\mathscr{L}_{c}^{\prime \prime}\right)$ can also be described in another way. As noted earlier, $\mathscr{L}_{c}^{\prime \prime} \cong \sum_{m \in \mathcal{J}}^{\oplus} \mathscr{Z}^{(m)}$, where $\mathscr{J} \subseteq\{1,2, \ldots, \infty\}$. However, it follows from multiplicity theory that any unitary $U$ which implements an automorphism of $\sum_{m \in \mathcal{F}}^{\oplus} \mathscr{Z}^{(m)}$ can be decomposed as a direct sum $\sum_{m \in \mathcal{G}}^{\oplus} U_{m}$ of unitaries $U_{m}$ which implement automorphisms of $\mathscr{Z}^{(m)}$. Consequently, $s$-Aut $\left(\mathscr{L}_{c}^{\prime \prime}\right) \cong \sum_{m \in \mathcal{I}}^{\oplus} s$-Aut $\left(\mathscr{Z}^{(m)}\right)$.

We turn now to derivations of $\mathscr{L}^{\prime}+\mathscr{K}$. A derivation of a Banach algebra $\mathfrak{A} \subseteq \mathscr{B}(\mathscr{H})$ is a linear operator $\delta: \mathfrak{A} \rightarrow \mathfrak{A}$ which satisfies the property $\delta(S T)=\delta(S) T+S \delta(T)$ for all $S, T \in \mathfrak{A} . \quad \delta$ is denoted by ad $X$ if $\delta(S)=X S-S X$ for some $X \in \mathscr{B}(\mathscr{H})$, and $\delta$ is inner if $\delta=\operatorname{ad} X$ for some $X \in \mathfrak{A}$. If $\delta$ is a continuous derivation, then $\delta$ is the infinitesimal generator of the uniformly continuous oneparameter automorphism group $\{\exp (t \delta): t \in \mathbb{R}\}$, and if $\delta=\operatorname{ad} X$, then $\exp (t \delta)=\operatorname{Ad}(\exp (t X))$. The approach taken here for derivations of essential commutants of abelian von Neumann algebras is similar to the one used in [DW, 3.11-3.13] for quasitriangular algebras (also see [W1] and [W2]). In the following, $\|T\|_{e}$ denotes the essential norm of $T$, i.e., $\|T\|_{e}=\|q(A)\|$, where $q$ is the canonical projection of $\mathscr{B}(\mathscr{H})$ onto the Calkin algebra $\mathscr{B}(\mathscr{H}) / \mathscr{K}(\mathscr{H})$.

Lemma 21. Suppose $\mathscr{L}$ is a CSL. If $\operatorname{Ad} V \in \operatorname{Aut}\left(\mathscr{L}^{\prime}+\mathscr{K}\right)$ and $\|V-I\|_{e}<\frac{1}{2}$, then $\operatorname{Ad} V$ is inner.

Proof. Without loss of generality, assume $\mathscr{L}$ is complemented, and apply Lemma 8 to obtain operators $U, P$, and $Q$. Let $\alpha=\operatorname{Ad} V$, and define $\widetilde{\Gamma}$ as in the proof of Corollary 20. Since $U-I \in \mathscr{K}$, we 
have $\|U V-I\|_{e}<\frac{1}{2}$ also, and it follows that

$$
\begin{array}{r}
\left\|U V T V^{*} U^{*}-T\right\|_{e} \leq \\
+U V T V^{*} U^{*}-T V^{*} U^{*} \|_{e} \\
+\left\|T V^{*} U^{*}-T\right\|_{e}<\|T\|_{e}
\end{array}
$$

for all $T \in \mathscr{B}(\mathscr{H})$. Now $(\operatorname{Ad} U V)\left(\mathscr{L}_{c}\right)=\mathscr{L}_{c}$ and $(\operatorname{Ad} U V)\left(\mathscr{L}_{a}^{P}\right)=$ $\mathscr{L}_{a}^{Q}$ with $($ Ad $U V)(P)=Q$. Therefore, $($ Ad $U V)(A)=A$ for all $A \in$ $\mathscr{L}_{c}$ since $U V A V^{*} U^{*}-A$ is the difference of two infinite commuting projections. Likewise, $(\operatorname{Ad} U V)\left(A P^{\perp}\right)=A Q^{\perp}$ for all $A \in \mathscr{I}(\mathscr{L})$. Thus, $\gamma(\alpha)=\mathrm{id}$ and $l_{\alpha}=\mathrm{id}$.

Let $\mathscr{P}=\{A \in \mathscr{F}(\mathscr{L}): A \leq P\}$ and $\mathscr{Q}=\{A \in \mathscr{F}(\mathscr{L}): A \leq Q\}$. Then Ad $U V$ is a bijection of $\mathscr{F}(\mathscr{L}) \backslash \mathscr{P}$ onto $\mathscr{F}(\mathscr{L}) \backslash \mathscr{Q}$. Suppose $(\operatorname{Ad} U V)\left(E_{i}\right) \neq E_{i}$ for an infinite set $\left\{E_{i}: 1 \leq i<\infty\right\} \subseteq \mathscr{F}(\mathscr{L}) \backslash \mathscr{P}$. Let $K$ be a compact operator such that $\|U V-I-K\|<\frac{1}{2}$, and let $e_{i} \in E_{i} \mathscr{H}$ with $\left\|e_{i}\right\|=1$. Then

$$
\left\|(U V-I-K)\left(e_{i}\right)\right\| \geq\left\|U V e_{i}-e_{i}\right\|-\left\|K e_{i}\right\| \geq \sqrt{2}-\left\|K e_{i}\right\|>\frac{1}{2}
$$

for $i$ large enough, since $\left\|K e_{i}\right\| \rightarrow 0$. This contradiction shows that there is a finite set $\widetilde{P} \supseteq \mathscr{P}$ such that $(\operatorname{Ad} U V)(A)=A$ for all $A \in$ $\mathscr{F}(\mathscr{L}) \backslash \widetilde{\mathscr{P}}$, and therefore, $\varphi_{\alpha}=[\mathrm{id}]$. The result now follows from Corollary 20 and the definition of $\widetilde{\Gamma}$.

THEOREM 22. If $\mathscr{L}$ is a CSL, then every derivation $\delta: \mathscr{L}^{\prime}+\mathscr{K} \rightarrow$ $\mathscr{L}^{\prime}+\mathscr{K}$ is inner.

Proof. The proof is essentially identical to [DW, Theorem 3.13]. Again, we can assume $\mathscr{L}$ is complemented. By [DW, Lemma 3.12], $\delta=\operatorname{ad} X$ for some $X \in \mathscr{B}(\mathscr{H})$. Then $\left\{\alpha_{t}=\exp (t \delta)=\operatorname{Ad}\left(e^{t X}\right): t \in\right.$ $\mathbb{R}\}$ is a uniformly continuous automorphism group of $\mathscr{L}^{\prime}+\mathscr{K}$, and $\left\|e^{t X}-I\right\|_{e}<\frac{1}{2}$ for $t$ sufficiently small. Thus, by Lemma 21, $\alpha_{t}$ is inner for $t$ small. It follows that $X=\lim _{t \rightarrow 0} t^{-1}\left(e^{t X}-I\right) \in \mathscr{L}^{\prime}+\mathscr{K}$, and therefore $\delta$ is inner.

Finally, we can make the following improvements to Lemma 21.

Corollary 23. Suppose $\mathscr{L}$ is a CSL and $\alpha \in \operatorname{Aut}\left(\mathscr{L}^{\prime}+\mathscr{K}\right)$.

(i) If the spectrum of $\alpha$ is contained in $\Omega=\{z \in \mathbb{C}: \operatorname{Re} z>0\}$, then $\alpha$ is inner.

(ii) If $\|\alpha-\mathrm{id}\|<1$, then $\alpha$ is inner. 
Proof. (i) follows from Theorem 22, using the argument given in [W2, Corollary 2.4]. (ii) follows from (i) since $\|\alpha-\mathrm{id}\|<1$ implies that the spectrum of $\alpha$ is contained in $\Omega$.

\section{REFERENCES}

[An] N. T. Andersen, Compact perturbations of reflexive algebras, J. Funct. Anal.. 38 (1980), 366-400.

[Ar] W. B. Arveson, Operator algebras and invariant subspaces, Ann. of Math., 100 (1974), 433-532.

[D1] K. R. Davidson, Nest Algebras: Triangular Forms for Operator Algebras on Hilbert Space, Wiley, New York, 1988.

[D2] - Similarity and compact perturbations of nest algebras, J. Reine Angew. Math., 348 (1984), 72-87.

[DW] K. R. Davidson and B. H. Wagner, Automorphisms of quasitriangular algebras, J. Funct. Anal., 59 (1984), 612-627.

[Di] J. Dixmier, Les algèbres d'opérateurs dans l'espace Hilbertien, GauthierVillars, Paris, 1969.

[JP] B. E. Johnson and S. K. Parrott, Operators commuting with a von Neumann algebra modulo the set of compact operators, J. Funct. Anal., 11 (1972), 39-61.

[KR] R. V. Kadison and J. R. Ringrose, Fundamentals of the Theory of Operator Algebras, Academic Press, Orlando, 1986.

[P] J. Plastiras, Compact perturbations of certain von Neumann algebras, Trans. Amer. Math. Soc., 234 (1977), 561-577.

[W1] B. H. Wagner, Automorphisms and derivations of certain operator algebras, Doctoral Dissertation, University of California, Berkeley.

[W2] _ Derivations of quasitriangular algebras, Pacific J. Math., 114 (1984), 243-255.

[W3] _-, Quasidiagonal operator algebras, Illinois J. Math., (to appear).

Received October 11, 1989.

IOWA STATE UnIVeRsity

AMES, IA 50011 


\section{PACIFIC JOURNAL OF MATHEMATICS EDITORS}

\author{
V. S. VARAdaraJan \\ (Managing Editor) \\ University of California \\ Los Angeles, CA 90024-1555-05 \\ Herbert Clemens \\ University of Utah \\ Salt Lake City, UT 84112 \\ THOMAS ENRIGHT \\ University of California, San Diego \\ La Jolla, CA 92093
}

R. FINN

Stanford University

Stanford, CA 94305

Hermann FlaschKa

University of Arizona

Tucson, AZ 85721

VAUGHAN F. R. Jones

University of California

Berkeley, CA 94720

STEVEN KerckHofF

Stanford University

Stanford, CA 94305

\section{C. MOORE}

University of California

Berkeley, CA 94720

Martin ScharlemanN

University of California

Santa Barbara, CA 93106

HAROLd STARK

University of California, San Diego

La Jolla, CA 92093
R. ARENS
E. F. BECKENBACH
F. WoLF
K. YoshidA
ASSOCIATE EDITORS

\begin{tabular}{ll}
\multicolumn{1}{c}{$(1906-1982)$} & \multicolumn{1}{c}{$(1904-1989)$} \\
SUPPORTING & INSTITUTIONS \\
UNIVERSITY OF ARIZONA & UNIVERSITY OF OREGON \\
UNIVERSITY OF BRITISH COLUMBIA & UNIVERSITY OF SOUTHERN CALIFORNIA \\
CALIFORNIA INSTITUTE OF TECHNOLOGY & STANFORD UNIVERSITY \\
UNIVERSITY OF CALIFORNIA & UNIVERSITY OF HAWAII \\
MONTANA STATE UNIVERSITY & UNIVERSITY OF TOKYO \\
UNIVERSITY OF NEVADA, RENO & UNIVERSITY OF UTAH \\
NEW MEXICO STATE UNIVERSITY & WASHINGTON STATE UNIVERSITY \\
OREGON STATE UNIVERSITY & UNIVERSITY OF WASHINGTON
\end{tabular}

The Supporting Institutions listed above contribute to the cost of publication of this Journal, but they are not owners or publishers and have no responsibility for its content or policies.

Mathematical papers intended for publication in the Pacific Journal of Mathematics should be in typed form or offset-reproduced (not dittoed), double spaced with large margins. Please do not use built up fractions in the text of the manuscript. However, you may use them in the displayed equations. Underline Greek letters in red, German in green, and script in blue. The first paragraph must be capable of being used separately as a synopsis of the entire paper. In particular it should contain no bibliographic references. Please propose a heading for the odd numbered pages of less than 35 characters. Manuscripts, in triplicate, may be sent to any one of the editors. Please classify according to the 1991 Mathematics Subject Classification scheme which can be found in the December index volumes of Mathematical Reviews. Supply name and address of author to whom proofs should be sent. All other communications should be addressed to the managing editor, or Elaine Barth, University of California, Los Angeles, California 90024-1555-05.

There are page-charges associated with articles appearing in the Pacific Journal of Mathematics. These charges are expected to be paid by the author's University, Government Agency or Company. If the author or authors do not have access to such Institutional support these charges are waived. Single authors will receive 50 free reprints; joint authors will receive a total of 100 free reprints. Additional copies may be obtained at cost in multiples of 50 .

The Pacific Journal of Mathematics (ISSN 0030-8730) is published monthly except for July and August. Regular subscription rate: $\$ 190.00$ a year (10 issues). Special rate: $\$ 95.00$ a year to individual members of supporting institutions.

Subscriptions, orders for numbers issued in the last three calendar years, and changes of address should be sent to Pacific Journal of Mathematics, P.O. Box 969, Carmel Valley, CA 93924, U.S.A. Old back numbers obtainable from Kraus Periodicals Co., Route 100, Millwood, NY 10546.

The Pacific Journal of Mathematics at P.O. Box 969, Carmel Valley, CA 93924 (ISSN 0030-8730) is published monthly except for July and August. Second-class postage paid at Carmel Valley, California 93924, and additional mailing offices. Postmaster: send address changes to Pacific Journal of Mathematics, P.O. Box 969, Carmel Valley, CA 93924.

PUBLISHED BY PACIFIC JOURNAL OF MATHEMATICS, A NON-PROFIT CORPORATION Copyright (C) 1991 by Pacific Journal of Mathematics 


\section{Pacific Journal of Mathematics}

Vol. 149, No. $2 \quad$ June, 1991

Manuel Alfaro Garcia, Mark Conger and Kenneth Hodges, The structure

of singularities in $\Phi$-minimizing networks in $\mathbf{R}^{2}$

Werner Balser, Dependence of differential equations upon parameters in

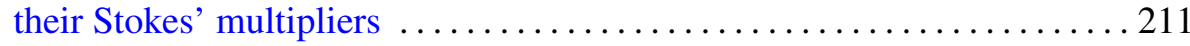

Enrico Casadio Tarabusi and Stefano Trapani, Envelopes of holomorphy

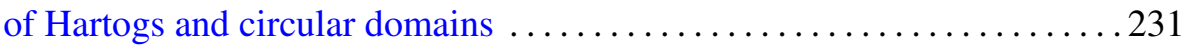

Hermann Flaschka and Luc Haine, Torus orbits in $G / P \ldots \ldots \ldots \ldots 251$

Gyo Taek Jin, The Cochran sequences of semi-boundary links .......... 293

Yasuyuki Kawahigashi, Cohomology of actions of discrete groups on

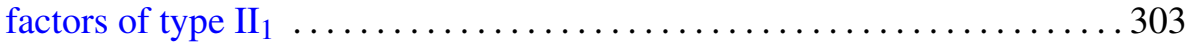

Ki Hyoung Ko and Lawrence Smolinsky, A combinatorial matrix in 3-manifold theory

W. B. Raymond Lickorish, Invariants for 3-manifolds from the combinatorics of the Jones polynomial

Peter Arnold Linnell, Zero divisors and group von Neumann algebras . . . . . 349

Bruce Harvey Wagner, Classification of essential commutants of abelian

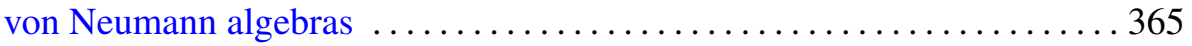

Herbert Walum, Multiplication formulae for periodic functions ..........383 